\title{
Clean CKM Information from $B_{d}(t) \rightarrow D^{(*) \mp} \pi^{ \pm}$
}

\author{
Isard Dunietz \\ Fermi National Accelerator Laboratory, P.O. Box 500, Batavia, IL 60510
}

(July 2, 2021)

\begin{abstract}
It has been known for many years that the $B_{d}(t) \rightarrow D^{(*) \mp}\left\{\pi^{ \pm}, \rho^{ \pm}, a_{1}^{ \pm}\right\}$ modes may involve observable $\mathrm{CP}$ violating effects. This note describes how to determine cleanly the Cabibbo-Kobayashi-Maskawa (CKM) phase $\phi=-2 \beta-\gamma=-\pi+\alpha-\beta$, even in the presence of possible final state interactions. A discrete ambiguity remains.
\end{abstract}




\section{INTRODUCTION}

The next decade will witness an unprecedented number of consistency checks on whether the CKM (Cabibbo-Kobayashi-Maskawa) hypothesis [1] correctly describes CP violation. While the gold-plated $B_{d} \rightarrow J / \psi K_{S}$ asymmetry [2] cleanly determines $\sin 2 \beta$, the other angles of the CKM unitarity triangle are harder to obtain [3].

Here we report on a clean method that extracts the CKM phase combination $2 \beta+\gamma$ or $\beta-\alpha$. While in principle all hadronic uncertainties can be disentangled [4, 5], in practice this is unfeasible for first generation experiments. Those experiments could determine the CKM phase, however, by incorporating related modes, as shown below. Since $\beta$ will be known, the CKM angle $\alpha$ or $\gamma$ could be obtained cleanly [up to a discrete ambiguity]. This is important, because accurate knowledge of the CKM parameters will constrain or rule out the CKM explanation for CP violation.

A time dependent study of $B_{d}(t) \rightarrow \pi^{+} \pi^{-}$is not capable of extracting the CKM parameter $\sin 2 \alpha$ cleanly, because of non-negligible penguin amplitudes. That can be inferred from the recent CLEO result for $\pi^{+} \pi^{-} / K^{+} \pi^{-}$[6], which indicates that penguin amplitudes are sizable in $B \rightarrow \pi \pi$ transitions. Thus, the clean determination of $\alpha$ from $B \rightarrow \pi \pi$ modes requires the study of $B_{d} \rightarrow \pi^{0} \pi^{0}$ [7] which is almost impossible at hadron accelerators [see, however, Ref. [8]], but may be possible at $\Upsilon(4 S)$ factories if the branching-ratio is not too small. Quinn and Snyder proposed to determine $\alpha$ from Dalitz plot analyses of $B_{d} \rightarrow \rho \pi[9]$. That method works if the non-resonant and other $B \rightarrow 3 \pi$ amplitudes are well understood, which may require large statistics [10]. Those modes involve $\pi^{0}$ 's and thus can be more naturally studied at present at $e^{+} e^{-}$colliders. Experiments at hadron accelerators would greatly enhance their $b$-physics reach by developing methods for efficient photon, $\pi^{0}, \eta, \eta^{\prime}$ reconstruction.

Denote the $B_{d} / \bar{B}_{d}$ modes $D^{(*)-}\left\{\pi^{+}, \rho^{+}, a_{1}^{+}, \ldots\right\}$ by $f$, and $\bar{f} \equiv \mathrm{CP} f$. Sachs stressed the importance of such non-leptonic, non-CP eigenstates in mixing-induced CP violation studies [11]. Until then, mixing-induced CP studies focused on either same-sign dilepton 
asymmetries [12] or on CP eigenmodes [2,13]. However, CP violation can also be seen [either time-dependent or time-integrated] with non-CP eigenstates [11, 114,[15]:

$$
\begin{aligned}
& \Gamma\left(B_{d}(t) \rightarrow f\right) \neq \Gamma\left(\bar{B}_{d}(t) \rightarrow \bar{f}\right), \\
& \Gamma\left(B_{d}(t) \rightarrow \bar{f}\right) \neq \Gamma\left(\bar{B}_{d}(t) \rightarrow f\right) .
\end{aligned}
$$

For instance, the $B_{d}(t) \rightarrow \bar{f}$ process involves the direct amplitude $B_{d} \rightarrow \bar{f}$ governed by the tiny CKM combination $V_{u b}^{*} V_{c d}$ and the mixing-induced amplitude $B_{d}(t) \rightarrow \bar{B}_{d} \rightarrow \bar{f}$, where the latter $\bar{B}_{d} \rightarrow \bar{f}$ transition is governed by the CKM favored $V_{c b} V_{u d}^{*}$ combination.f The disparate strengths of the two interfering amplitudes cause the CP asymmetry to be at the few percent level. The CP asymmetry is larger for process (1.2) than for process (1.1), because the two interfering amplitudes are made significantly less disparate in size by the judicious positioning of the small $B_{d}-\bar{B}_{d}$ mixing-amplitude.

Since the distinction between an initially unmixed $B_{d}$ and $\bar{B}_{d}$ (flavor-tagging) entails normally some impurity, one will have to correct for a (serious) asymmetric background $\left[\bar{B}_{d}(t) \rightarrow \bar{f}\right]$. The correction is, however, well understood because it depends on the same observables (see Eq. (11.5) below).

The interference term is [4]

$$
\lambda \equiv \frac{q}{p} \frac{\left\langle f \mid \bar{B}_{d}\right\rangle}{\left\langle f \mid B_{d}\right\rangle}=\rho e^{i(\phi+\Delta)},
$$

where $\rho$ denotes the magnitude of the amplitude ratio. The weak phase (CKM phase) is

$$
\phi=-2 \beta-\gamma=-\pi+\alpha-\beta,
$$

and $\Delta$ denotes a possible strong phase difference. Ref. [4] demonstrated how a timedependent study of the four rates,

$$
\Gamma\left(B_{d}(t) \rightarrow f\right), \Gamma\left(B_{d}(t) \rightarrow \bar{f}\right), \Gamma\left(\bar{B}_{d}(t) \rightarrow \bar{f}\right), \Gamma\left(\bar{B}_{d}(t) \rightarrow f\right),
$$

\footnotetext{
*Those CKM combinations are unique and the same for either the color-allowed spectator graph or the internal- $W$ graph, predicted to be much smaller.
} 
extracts the three observablest

$$
\rho, \sin (\phi+\Delta), \sin (\phi-\Delta)
$$

The weak phase $\phi$ can be determined up to a discrete ambiguity from fundamental trigonometry. By the time such demanding studies can be performed, the angle $\beta$ will be well known from the $B_{d} \rightarrow J / \psi K_{S}$ asymmetry. Thus the angle $\alpha$ (or $\left.\gamma\right)$ can be cleanly extracted, because penguin amplitudes cannot contribute. A discrete ambiguity remains.f

While our observations are true in principle, it is exceedingly difficult, in practice, to fit for such small $\rho$ parameters in time-dependent studies. We therefore suggest to determine $\rho$ elsewhere. The observable $\rho^{2}$ is essentially the ratio of rates,

$$
\rho^{2}=\frac{\Gamma\left(\bar{B}_{d} \rightarrow D^{(*)-}\left\{\pi^{+}, \rho^{+}, a_{1}^{+}, \ldots\right\}\right)}{\Gamma\left(B_{d} \rightarrow D^{(*)-}\left\{\pi^{+}, \rho^{+}, a_{1}^{+}, \ldots\right\}\right)} .
$$

The difficulty in obtaining $\rho^{2}$ lies in determining the tiny numerator. That numerator can be obtained by studying the (a) $\bar{B}_{s} \rightarrow D^{(*)-} K^{(*)+}$ processes, (b) $\bar{B}_{d} \rightarrow D_{s}^{(*)-}\left\{\pi^{+}, \rho^{+}, a_{1}^{+}, \ldots\right\}$ processes [where the strangeness content of the final state automatically tags the $\bar{B}$ flavor at time of decay], (c)

$$
\rho^{2} \approx \frac{2 \Gamma\left(B^{-} \rightarrow D^{(*)-}\left\{\pi^{0}, \rho^{0}, a_{1}^{0}, \ldots\right\}\right)}{\Gamma\left(B_{d} \rightarrow D^{(*)-}\left\{\pi^{+}, \rho^{+}, a_{1}^{+}, \ldots\right\}\right)} .
$$

Small corrections to the approximations can be incorporated once they have been investigated experimentally and theoretically [18].

Furthermore, it is probable that the strong phase difference is small $\Delta \approx 0 \bmod \pi$, $\beta$ and a first generation experiment may wish to fit the four time-dependent rates for the single

\footnotetext{
${ }^{\dagger}$ For a non-vanishing $B_{d}-\bar{B}_{d}$ width difference $\Delta \Gamma$ (expected to be at the $1 \%$ level [16]), the relevant observables can be extracted from fits to more involved time-dependences [17]. Of course, the accurate extraction of the CKM angle $\beta$ will also involve more elaborate fits.

${ }^{\ddagger}$ It maybe partially resolved because $\phi$ is the same for the various modes, whereas $\Delta$ could be mode-dependent.
}

$\S$ Both interfering amplitudes are governed dominantly by color-allowed spectator graphs. Under 
parameter $\phi$. To determine $\sin \phi$ to an accuracy of \pm 0.1 , one requires about $10^{8}$ (fully) flavor-tagged $B_{d}+\bar{B}_{d}$ mesons. This estimate assumes $\mathcal{O}(1)$ detection efficiencies and a nominal $B\left(B_{d} \rightarrow f\right)=0.003$. The relevant branching-ratios $B\left(B_{d} \rightarrow f\right)$ are indeed large $\mathcal{O}(0.003-0.01)$ [19]. The main difficulty lies in accurately observing the small asymmetry governed by the interference term $\lambda$, whose magnitude is approximately

$$
\rho \approx\left|V_{u b} / V_{c b}\right| \times\left|V_{c d} / V_{u d}\right| \approx 0.02
$$

We anticipate that the observable $\rho^{2}$ will be known to sufficient accuracy, because several $\rho^{2}$ extractions do not require flavor-tagging and could infer $\rho^{2}$ from less CKM suppressed transitions (as outlined above).

The unique kinematics of the $D^{* \pm} \pi^{\mp}$ mode may permit a semi-inclusive reconstruction by using the soft $\pi^{ \pm}$in the $D^{* \pm} \rightarrow \pi^{ \pm} \stackrel{(-)}{D^{0}}$ decay [20]. Those modes are also a natural for detectors operating at hadron accelerators, because the analogous $B_{s} \rightarrow D_{s}^{-} \pi^{+}, D_{s}^{-} \pi^{+} \pi^{-} \pi^{+}$ processes, important for $\Delta m_{s}$ measurements, were shown to be accessible with large rates. Further note that the $B_{d} \rightarrow D^{* \mp}\left\{\rho^{ \pm}, a_{1}^{ \pm}\right\}$modes allow also the clean determination of the CKM phase $\phi$, by employing angular correlations. Those angular correlations permit the study of more involved CP violating observables.

In conclusion, this note demonstrates that the $B_{d} \rightarrow D^{(*) \mp}\left\{\pi^{ \pm}, \rho^{ \pm}, a_{1}^{ \pm}, \ldots\right\}$ transitions allow the clean extraction of the CKM angle $\phi=-2 \beta-\gamma=-\pi+\alpha-\beta$. That may play a role in constraining or ruling out the CKM hypothesis for CP violation.

\section{ACKNOWLEDGEMENTS}

We are grateful to R. Fleischer and C. Quigg for informing us that the BABAR collaboration is studying the feasibility of such modes by semi-inclusive reconstruction. We thank

the factorization approximation no final state phase difference occurs. Such phase differences could be generated by non-factorizable contributions, rescatterings and subleading diagrams as catalogued in Ref. [18], and are all expected to be small. 
J. Lewis, J.L. Rosner, M. Shapiro and S. Stone for discussions. This work was supported in part by the Department of Energy, Contract No. DE-AC02-76CH03000. 


\section{REFERENCES}

[1] N. Cabibbo, Phys. Rev. Lett. 10 (1963) 531; M. Kobayashi and T. Maskawa, Prog. Theor. Phys. 49 (1973) 652.

[2] I.I. Bigi and A.I. Sanda, Nucl. Phys. B193 (1981) 85.

[3] For a recent review see, for instance, A.J. Buras and R. Fleischer, to appear in Heavy Flavours II, World Scientific (1997), eds. A.J. Buras and M. Linder, April 1997 hepph/9704376].

[4] R. Aleksan, I. Dunietz and B. Kayser, Z. Phys. C54 (1992) 653.

[5] J.P. Silva, hep-ph/9711412.

[6] CLEO Collaboration, CLEO-CONF 97-24 (EPS-334), August 1997.

[7] M. Gronau and D. London, Phys. Rev. Lett. 65 (1990) 3381.

[8] I. Dunietz, in Proceedings of the Workshop on B Physics at Hadron Accelerators, Snowmass, Colorado, June 21 - July 2, 1993, p. 83, ed. by Patricia McBride and C. Shekhar Mishra.

[9] A.E. Snyder and H.R. Quinn, Phys. Rev. D48 (1993) 2139.

[10] Francois Le Diberder and Sophie Versillé, private communication.

[11] R.G. Sachs, Enrico Fermi Institute Report, EFI-85-22-CHICAGO, February 1985 (unpublished). Revised manuscript published as I. Dunietz and R.G. Sachs, Phys. Rev. D37 (1988) 3186; (E) ibid. D39 (1989) 3515.

[12] A. Pais and S.B. Treiman, Phys. Rev. D12 (1975) 2744; T. Altomari, L. Wolfenstein and J.D. Bjorken, Phys. Rev. D 37 (1988) 1860; M. Lusignoli, Z. Phys. C41 (1989) 645

[13] L. Wolfenstein, Nucl. Phys. B246 (1984) 45.

[14] I. Dunietz and J.L. Rosner, Phys. Rev. D34 (1986) 1404. 
[15] D. Du, I. Dunietz and Dan-di Wu, Phys. Rev. D34 (1986) 3414.

[16] M. Beneke, G. Buchalla and I. Dunietz, Phys. Rev. D54 (1996) 4419.

[17] I. Dunietz, Phys. Rev. D52 (1995) 3048.

[18] M. Gronau, O.F. Hernandez, D. London and J.L. Rosner, Phys. Rev. D52 (1995) 6356.

[19] R.M. Barnett et al. (Particle Data Group), Phys. Rev. D54 (1996) 1.

[20] CLEO Collaboration, CLNS 97/1485, CLEO 97-11, hep-ex/9706019 v2, December 1997. 